\title{
EDITORIAL
}

\section{Recent progress in physics beyond the standard model}

\author{
Shou-Hua Zhu \\ Institute of Theoretical Physics, Peking University, Beijing 100871, China \\ E-mail: shzhu@pku.edu.cn \\ Received April 11, 2013; accepted April 17, 2013
}

Physics beyond the standard model (BSM) is one of the most important topics in high energy physics. 2012 is marked as a special year for BSM. For example, in March, Daya Bay collaboration found that the last mixing angle $\theta_{13}$ for neutrino is not 0 . In July, the standard model (SM) like Higgs boson was discovered at the Large Hadron Collider (LHC). BSM was the hottest topic at 20th International Conference on Supersymmetry and Unification of Fundamental Interactions (SUSY2012), which was held in August in Peking University.

We invited several SUSY2012 speakers to review the latest progress of BSM based on their talks at the conference. The topics include both the experimental [1-4] and theoretical [5, 6] progresses on neutrino, Higgs boson, supersymmetry and other BSM.

Obviously there are still open questions for BSM, which could be answered in the next decade. We name a few:

- Whether the newly discovered SM-like Higgs boson is really the SM one?

- Whether there are more Higgs bosons?

- Whether there are supersymmetric partners at TeV scale?

- Whether one can discover dark matter at TeV scale?

- Whether there are more space-time dimensions?

- Whether there are new CP violations, which can account for the matter-dominant Universe?

With the development of BSM, the frontier knowledge of mankind will be definitely broadened and deepened.

\section{References}

1. M. He, Front. Phys., 2013, 8(3): 242

2. P. Pralavorio [on behalf of the ATLAS Collaboration], Front. Phys., 2013, 8(3): 248

3. A. Perieanu [on behalf of the CMS Collaboration], Front.
Phys., 2013, 8(3): 257

4. G. J. Davies [on behalf of the CDF and D0 Collaborations], Front. Phys., 2013, 8(3): 270

5. J. Chang, K. M. Cheung, P.-Y. Tseng, and T.-C. Yuan, Front. Phys., 2013, 8(3): 285

6. P. Nath, Front. Phys., 2013, 8(3): 294 